\title{
Operators induced by transformations of Gaussian variables
}

\author{
by W. MLAK (Kraków)
}

Franciszek Leja in memoriam

\begin{abstract}
The paper deals with composition operators $T_{a}: f \rightarrow f \circ a(a: R \rightarrow R)$ in the space $L^{2}\left(\frac{1}{\sqrt{2 \pi}} e^{-x^{2} / 2} d x\right)$ and tensor products as well some unitarily equivalent operators of $T_{a}-s$.

The present paper is partly expository. We point out some relations between several models and interpretations of operators in question. Some applications to certain integral equations are presented. We are interested mostly in pointing out the basic ideas and do not formulate the whole of more or less simple consequences.
\end{abstract}

1. In all what follows we consider merely Borel measurable functions. The reason is that the class of such functions is closed with respect to the operation of composition of functions. The classical examples for Lebesgue measurable functions show, that the composition of two such functions need not be a Lebesgue measurable function. It follows then that if we consider a class of functions on $R=(-\infty,+\infty)$ and the map $\left(T_{a} f\right)(x)=f(a(x))$ for $f$ and a defined on $R(a: R \rightarrow R)$, then $T_{a}$ preserves the measurability, if both $f$ and $a$ are Borel measurable functions; this is not the case in the class of Lebesgue measurability, i.e. if we allow $f$ and $a$ to be Lebesgue measurable. Since we need a nicely defined $T_{a}$, we define the Hilbert space

$$
L^{2}(p)=L^{2}\left(\frac{1}{\sqrt{2 \pi}} e^{-x^{2} / 2} d x\right)
$$

as the space of Borel measurable complex functions on $R$, with square summable modulus with respect to the measure

$$
d p=\frac{1}{\sqrt{2 \pi}} e^{-x^{2} / 2} d x
$$


defined on Borel subsets of $\boldsymbol{R}$, with the natural identification of two functions which differ on a Borel set of zero Lebesgue measure $m$.

This identification requires that a should satisfy the following condition:

$a$ is Borel measurable and $p\left(a^{-1}(\sigma)\right)=0$ if $p(\sigma)=0$ (for each Borel set $\sigma \subset R)$; see [5], Chapter 8,5.

Notice that the following condition holds true:

If $a: R \rightarrow R$ is strictly monotonic and $a^{-1}$; the inverse of $a$ is absolutely continuous (i.e. absolutely continuous on each finite interval $[\alpha, \beta] \subset a(R))$, then $a$ satisfies $(1.0)$.

The requirement of absolute continuity of $a^{-1}$ is needed, because in general, even if $a$ is absolutely continuous and strictly increasing, $a^{-1}$ need not be absolutely continuous.

If $c: R \rightarrow R$ is increasing strictly and continuous and maps p-zero Borel sets onto p-zero Borel sets, then $c$ is absolutely continuous (Banach-Zarecky theorem). Thus the absolute continuity in (1.1) is needed essentially in order to conclude that (1.1) implies in this case (1.0). The non-monotonic function $a$ which satisfies (1.0) is for example $a(x)=|x|$. Going back to the operator $T_{a}$ within $L^{2}(p)$ we will recall the following property - see [5], Chapter 8,5:

Suppose a satisfies (1.0). Let $D=\left\{f \in L^{2}(p): f \circ a \in L^{2}(p)\right\}$. Then the operator $T_{a}: D \rightarrow L^{2}(p)$ defined by formula $\left(T_{a} f\right)(x)=f(a(x))$ for $p$ almost all $x$ is closed.

Proof. Suppose $T_{a} f_{n} \rightarrow g, f_{n} \rightarrow f$ in $L^{2}(p)$ for $f_{n} \in L^{2}(p)$. There is a subsequence $\left\{n_{k}\right\}, n_{k \rightarrow \vec{k} \rightarrow \infty}$, such that $\left(T_{a} f_{n_{k}}\right)(x)=f_{n_{k}}(a(x)) \rightarrow g(x)$ for $x \in$ $R-\alpha$, where $p(\alpha)=0$ and $f_{n_{k}}(y) \rightarrow f(y)$ for $y \in R-\beta$, where $p(\beta)=0$. It follows that if $x \notin a^{-1}(\beta)$ and $x \in \alpha$, then $g(x)=f(a(x))$. Since $p\left(a^{-1}(\beta)\right)=0$ and $p(\alpha)=0$, then $g(x)=f(a(x))$ for $p$-a.e. as was to be proved.

Notice that the operator $T_{a}$ of (1.2) is maximal in the sense, that when we define $\left(T_{a} f\right)(x)=f(a(x))$ on a dense manifold of $L^{2}(p)$, then $T_{a}$ is closable and its closure is included in $T_{a}$ of (1.2). This is the case for instance if

$\int_{-\infty}^{+\infty}|a(x)|^{2 k} e^{-x^{2} / 2} d x<+\infty$ for all $k=1,2, \ldots$ and $\left(\tilde{T}_{a} f\right)(x)=f(a(x))$ for $f \in \boldsymbol{P}=$ the linear manifold of all complex polynomials on $\boldsymbol{R}$. The following example illustrates (1.2):

EXAmple 1.0. Assume that $a$ is absolutely continuous on $R$ and 0 $<p_{a} \leqslant a^{\prime}(x) \leqslant q_{a}<+\infty$ for almost all $x$ and $a(0)=0$. It follows that $b$ $=a^{-1}$ is Lipschitzian and increases strictly. Moreover, $-|a(x)|^{2} \leqslant-p_{a}|x|^{2}$ for all $x$ and consequently $\lim a(x)= \pm \infty$. Moreover, $b^{\prime}(y)$ exists $p$-a.e. 
and $0<b^{\prime}, s_{a}=1 / q_{a} \leqslant b^{\prime}(y) \leqslant 1 / p_{a}, p$-a.e. Hence $-|b(y)|^{2} \leqslant-s_{a}|y|^{2}$ and consequently

$$
\begin{aligned}
& \int_{-\infty}^{+\infty}|a(x)|^{2 k} e^{-x^{2} / 2} d x=\int_{-\infty}^{+\infty}|y|^{2 k} b^{\prime}(y) e^{-|b(y)|^{2} / 2} d y \\
& \leqslant \frac{1}{p_{a}} \int_{-\infty}^{+\infty}|y|^{2 k} e^{-\left(s_{a} / 2\right) y^{2} d y}<+\infty \quad \text { for all } k=1,2,3, \ldots
\end{aligned}
$$

In previous notations $T_{a}$ and $\tilde{T}_{a}$ make now sense.

Let us notice finally that for pretty nice functions $a$ the operator $T_{a}$ of (1.2) need not be extendable to $a$ bounded one. Indeed, take $a(x)=2 x$ and the function $f(x)=e^{x^{2} / 8}$. It is plain that $f \in L^{2}(p)$, but

$$
\int_{-\infty}^{+\infty}|f(2 x)|^{2} e^{-x^{2} / 2} d x=+\infty
$$

as was to be proved.

All we have done above may be interpreted in terms of the simplest elementary Gaussian random variable $\xi(x)=x$, the probability measure being equal to $d p=\frac{1}{\sqrt{2 \pi}} e^{-x^{2} / 2} d x$. The operator $T_{a}$ is induced by a transformation $\xi \rightarrow a(\xi)$ of this random variable. Moreover, the whole space of all second order variables for $p$ is just $L^{2}(p)=$ the $L^{2}(p)$ closure of $P$.

2. This section deals with bounded $T_{a}$. We start with the following definition:

Definition 2.0. We say that the function $a: R \rightarrow R$ is of class $A_{+}$if it is absolutely continuous and satisfies the following conditions:

(i) $\quad a(0)=0$;

(ii) $0<p_{a} \leqslant a^{\prime}(x) \leqslant q_{a} \leqslant 1$ for almost all $x$ for constants $p_{a}, q_{a}$.

It follows from (ii) that $a$ is strictly increasing. Also $x_{2}-x_{1} \leqslant q_{a}\left(a\left(x_{2}\right)-\right.$ $\left.-a\left(x_{1}\right)\right)$ if $x_{1}<x_{2}$. Let $a^{-1}=b$. It follows that $b$ satisfies the Lipschitz condition, and consequently $b$ is absolutely continuous and

(iii) $\quad 0<1 / q_{a} \leqslant b^{\prime}(y) \leqslant 1 / p_{a}$ for almost all $y$.

Using arguments similar to that ones in Example 1.0 we conclude that for $a \in A_{+}, b=a^{-1}$ we have:

$$
\begin{gathered}
\lim _{x \rightarrow \pm \infty} a(x)= \pm \infty \\
-|b(y)|^{2} \leqslant-|y|^{2} \quad \text { for almost all } y \\
\lim _{y \rightarrow \pm \infty} b(y)= \pm \infty
\end{gathered}
$$


We notice that $A_{+}$is a unital semi-group with respect to compositions, i.e. if $a, c \in A_{+}$then $d=a \circ c \in A_{+}$. That $d$ is absolutely continuous follows from the absolute continuity of $c$ and the property that $a$ is Lipschitzian. The unit $e$ in $A_{+}$is the identity function $e(x)=x$.

TheOREM 2.0. Suppose that $a \in A_{+}$. Then the map $T_{a}: f \rightarrow f \circ a$ is a well defined bounded operator within the space $L^{2}(p)$ and $\left\|T_{a}\right\| \leqslant 1 / \sqrt{s(a)}$, where $s(a)=\inf _{R}$ ess $a^{\prime}$.

Proof. It is plain that for $f \in L^{2}(p)$ the function $f(a(x))$ is Borel measurable. Next, taking $b=a^{-1}$ and making the change of variables $x=b(y)$ we get by (iii), (iiv), (iiiv) that

$$
\begin{aligned}
\frac{1}{\sqrt{2 \pi}} \int_{-\infty}^{+\infty}|f(a(x))|^{2} e^{-x^{2} / 2} d x & =\frac{1}{\sqrt{2 \pi}} \int_{-\infty}^{+\infty}|f(y)|^{2} b^{\prime}(y) e^{-|b(y)|^{2} / 2} d y \\
& \leqslant\|f\|^{2} \sup _{R} \operatorname{ess} b^{\prime} \leqslant \frac{1}{\sqrt{2 \pi}} \frac{1}{p_{a}} \int_{-\infty}^{+\infty}|f(y)|^{2} e^{-y^{2} / 2} d y .
\end{aligned}
$$

Since $T_{a}$ is linear, we conclude that it is a bounded operator in $L^{2}(p)$ and $\left\|T_{a}\right\|^{2} \leqslant \sup _{R}$ ess $b^{\prime}=1 /$ inf ess $a^{\prime}$. Since in (ii) we can take $p_{a}=s(a)$, then by (iii) we can conclude that supess $b^{\prime} \leqslant 1 / s(a)$ which completes the proof of our assertion.

Corollary 2.0. Let $A_{-}$be the totality of all absolutely continuous functions $c: R \rightarrow R$ which satisfy the following conditions:

(i) $c(0)=0$;

(ii) $-1 \leqslant r_{c} \leqslant c^{\prime}(x) \leqslant m_{c}<0$ for almost all $x$ and some constants $r_{c}, m_{c}$.

It is plain that if $c \in A_{-}$then $a(x)=c(-x)$ is in $A_{+}$. Let $V$ be the unitary map in $L^{2}(p)$ defined by formula $(V g)(x)=g(-x)$. Then since $\left(T_{a} f\right)(x)=f(a(x))$ $=f(c(-x))$ then $\left(V T_{a} f\right)(x)=f(c(x))=\left(T_{c} f\right)(x)$, i.e. $V T_{a}=T_{c}$.

It follows now from Theorem 2.0 that $T_{c}$ is a well defined bounded operator in $L^{2}(p)$ and $\left\|T_{c}\right\|^{2} \leqslant 1 / s(a)$. The definition of $a$ implies that $s(a)$ $=-$ supess $c^{\prime}$. Notice yet that $A_{-}$is not closed under composition of its elements, but $A_{0}=A_{+} \cup A_{-}$is. It follows that the map $\varphi: A_{0} \rightarrow \mathscr{L}\left(L^{2}(p)\right)$ (= the algebra of all linear bounded operators in $L^{2}(p)$ ) defined by formula $\varphi(a)=T_{a}$ is a unital semi-group homomorphism; its restriction to $A_{+}$is also a unital homomorphism.

Let us define $A^{+}\left(A^{-}\right.$, respectively) the subset of $A_{+}\left(A_{-}\right.$, respectively) of all linear maps in $A_{+}\left(A_{-}\right)$, i.e. the totality of functions $\tilde{a}(x)=a x$, where $0<a \leqslant 1(-1 \leqslant a<0$, respectively). 
Proposition 2.0. If $\tilde{a} \in A^{+} \cup A^{-}$, then $\left\|T_{a}\right\|=1 / \sqrt{|a|}$, where $\tilde{a}(x)=a x$.

Proof. We conclude from Theorem 2.0 that $\left\|T_{a}\right\| \leqslant 1 / \sqrt{|a|}$. On the other hand, by general theorem (see [5], Chapter 8.5)

$$
\sup _{p(\sigma)>0} \frac{p\left(\tilde{a}^{-1}(\sigma)\right)}{p(\sigma)}=\left\|T_{\tilde{a}}\right\|^{2}
$$

for Borel sets $\sigma$. We take $x>0$ and $\sigma_{x}=[0, x]$. Then, for $a>0$,

$$
p\left(\sigma_{x}\right)=\frac{1}{\sqrt{2 \pi}} \int_{0}^{x} e^{-s^{2} / 2} d s \text { and } p\left(\tilde{a}^{-1}\left(\sigma_{x}\right)\right)=\frac{1}{\sqrt{2 \pi}} \int_{0}^{x / a} e^{-s^{2} / 2} d s .
$$

Now

$$
\frac{p\left(\tilde{a}^{-1}\left(\sigma_{x}\right)\right)}{p\left(\sigma_{x}\right)}=\frac{\int_{0}^{x / a} e^{-s^{2} / 2} d s}{\int_{0}^{x} e^{-s^{2} / 2} d s} \rightarrow \frac{1}{a}
$$

by de L'Hôspital rule, which completes the proof.

Corollary 2.1. If $a \in A_{0}$ is continuously differentiable in a neighbouhood of $x=0$ and $\left|a^{\prime}(x)\right| \geqslant\left|a^{\prime}(0)\right|>0$ for almost all $x$, then $\left\|T_{a}\right\|=1 / \sqrt{\left|a^{\prime}(0)\right|}$ for this being the case we can use the de L'Hôspital rule.

3. Suppose the functions $a_{1}, a_{2}, \ldots, a_{n}$ belong to $A_{+}$. Then by Theorem 2.0 the operators $T_{a_{k}}(k=1, \ldots, n)$ are bounded. Let $T_{n}=T_{a_{1}} \otimes T_{a_{2}} \otimes \ldots \otimes T_{a_{n}}$. $T_{n}$ is defined on the $n$-fold tensor power of the space $L^{2}(p)$, i.e. on

$$
L^{2}(p)^{\otimes^{n}}=L^{2}\left(p_{1}\right) \otimes L^{2}\left(p_{2}\right) \otimes \ldots \otimes L^{2}\left(p_{n}\right), \quad \text { where } d p_{k}=\frac{1}{\sqrt{2 \pi}} e^{-x_{k}^{2} / 2} d x_{k} .
$$

The last tensor product is just the space $L^{2}\left(p^{\otimes^{n}}\right)$, where $p^{\otimes^{n}}$ $=p_{1} \otimes p_{2} \otimes \ldots \otimes p_{n}$, because it is spanned by functions products $f_{1}\left(x_{1}\right) f_{2}\left(x_{2}\right) \ldots f_{n}\left(x_{n}\right)\left(f_{k} \in L^{2}\left(p_{k}\right)\right)$ which are identified with tensor products $f_{1} \otimes f_{2} \otimes \ldots \otimes f_{n}$. The space $L^{2}\left(p^{\otimes n}\right)$ is the Hilbert space of Borel measurable functions on $R^{n}$ with the inner product

$$
\begin{gathered}
(f, g)=\frac{1}{(\sqrt{2 \pi})^{n}} \int_{R^{n}} f(x) \overline{g(x)} e^{-|x|^{2 / 2} d x} \\
\left(|x|^{2}=\sum_{j \mid 1}^{n} x_{j}^{2}, x=\left(x_{1} \ldots x_{n}\right), d x=d x_{1} \ldots d x_{n}\right) .
\end{gathered}
$$

With these indentifications the operator $T_{a}$ is defined by formula.

$$
\left(T_{a} f\right)\left(x_{1} \ldots x_{n}\right)=f\left(a_{1}\left(x_{1}\right) \ldots a_{n}\left(x_{n}\right)\right) .
$$


This formula makes sense because the map $a: x=\left(x_{1} \ldots x_{n}\right)$ $\rightarrow\left(a_{1}\left(x_{1}\right) \ldots a_{n}\left(x_{n}\right)\right)$ is invertible, maps all Borel sets $B\left(R^{n}\right)$ in $R^{n}$ on $B\left(R^{n}\right)$ $=$ the totality of all Borel sets in $R^{n}$ (each $a_{k}$ is invertible and homeomorphism within $\left.R^{1}\right)$ and if $m_{n}(\sigma)=0\left(m_{n}=\right.$ the Lebesgue measure in $B\left(R^{n}\right)$ ), then $m_{n}\left(a^{-1}(\sigma)\right)=0$. Indeed, taking $b_{k}=a_{k}^{-1}$ and $b\left(x_{1} \ldots x_{n}\right)$ $=\left(b_{1}\left(x_{1}\right), \ldots, b_{n}\left(x_{n}\right)\right)$ and $\tilde{p}_{n}(\sigma)=\left(p_{1} \otimes p_{2} \otimes \ldots p_{n}\right)(b(\sigma))$ we have $\tilde{p}_{n} \equiv p^{\otimes^{n}}$ and $d \tilde{p}_{n} / d p>0 m_{n}$-almost everywhere.

Summing up, and using the Brown-Pearcy theorem [4], we get the following theorem:

TheOREM 3.0. Suppose that $a_{k} \in A_{+}$for $k=1,2, \ldots, n$. Then the operator $T_{a}=T_{a_{1}} \otimes \ldots \otimes T_{a_{n}}$ in $L^{2}\left(p^{\otimes n}\right)$ is well defined by formula

$$
\left(T_{a} f\right)\left(x_{1} \ldots x_{n}\right)=f\left(a_{1}\left(x_{1}\right) \ldots a_{n}\left(x_{n}\right)\right)
$$

and $\left\|T_{a}\right\|=\prod_{k \mid 1}^{n}\left\|T_{a_{k}}\right\| \leqslant \prod_{k \mid 1}^{n} \frac{1}{\sqrt{s\left(a_{k}\right)}}$ and the spectrum $\operatorname{Sp}\left(T_{a}\right)$ equals to $\prod_{k \mid 1}^{n} \operatorname{Sp}\left(T_{a_{k}}\right)$ $=\left\{z: z=z_{1} \cdot z_{2} \ldots z_{n}, z_{i} \in \operatorname{Sp}\left(T_{i}\right)\right.$ for $\left.i=1, \ldots, n\right\}$.

Suppose now that we given an infinite sequence $a_{1}, a_{2}, \ldots$ of functions belonging to $A_{+}$. For each $n$ we can construct the operator $T_{n}$. The natural question arises, namely in which space some limit of $T_{n}$ exists if $n$ tends to infinity. In order to avoid the general "tensoring" theory of infinite sequences of Hilbert spaces as well of infinite tensor products of operators in such spaces, we will show that in our situation some natural candidates for these objects can be directly constructed.

To begin with, notice first that the sequence $a_{k}, a_{k} \in A_{+}$defines a natural map $a$, namely that one coordinatewise mapping, i.e. $a$ maps $\left(x_{1}, x_{2}, \ldots, x_{n}, \ldots\right)$ on the infinite sequence $\left(a_{1}\left(x_{1}\right), a_{2}\left(x_{2}\right), \ldots, a_{n}\left(x_{n}\right), \ldots\right)$. The natural candidate of a space, in which $a$ can be considered is the classical Fréchet space $R^{x}$, the countable product of copies of $R^{1}$ equipped with the coordinatewise topology defined by metric

$$
(x, y)=\sum_{n \mid 1}^{\infty} \frac{1}{2^{n}} \frac{\left|x_{n}-y_{n}\right|}{1+\left|x_{n}-y_{n}\right|}
$$

for $x=\left(x_{1}, x_{2}, \ldots, x_{n}, \ldots\right), y=\left(y_{1}, y_{2}, \ldots, y_{n}, \ldots\right)$.

Let $B\left(R^{x}\right)$ be the $\sigma$-field of Borel sets in $R^{\infty}$ with the above metric. $B\left(R^{\infty}\right)$ is generated by cylinder sets, namely by sets $\underset{n \mid 1}{\mathrm{X}} \sigma_{n}, \sigma_{n} \in B\left(R^{1}\right)$ ( $=-$ the $\sigma$-field of Borel sets in $R^{1}$ ) where only a finite number of $\sigma_{n}$ is different from $R^{1}$. That such a $\sigma$-field is included in $B\left(R^{x}\right)$ is obvious. In fact $B\left(R^{\infty}\right)$ is generated by half planes $\pi_{u, c}=\left\{x \in R^{\infty}: u(x) \leqslant c\right\}$ (c real), where $u$ runs over the complete set of continuous linear functionals on $R^{\infty}$ (see [10], Chapter I, 9.1). Since every continuous linear functional $u$ is of the form $u(x)$ $=\sum_{i \mid 1}^{m(u)} u_{i} x_{i}, n(u)<+\infty$, and $\pi_{u, c}$ is a cylinder set, we get what has been to be 
proved. Next, if $a_{k} \in A_{+}$then the map $a:\left(x_{1}, x_{2}, \ldots, x_{n}, \ldots\right)$ $\rightarrow\left(a_{1}\left(x_{1}\right), a_{2}\left(x_{2}\right), \ldots, a_{n}\left(x_{n}\right), \ldots\right)$ is a homeomorphism of $R^{\infty}$ onto itself, so a $\left(B\left(R^{\infty}\right)\right)=B\left(R^{\infty}\right)$, i.e. a maps Borel sets in $R^{\infty}$ onto Borel sets as well its inverse $a^{-1}$ does the same, i.e. $a$ and $b=a^{-1}$ are both Borel measurable.

Let $p_{k}$ be a copy of the Gaussian measure on $R^{1}$ written as $d p_{k}$ $=\frac{1}{\sqrt{2 \pi}} e^{-x_{k}^{2} / 2} d x_{k}$. The Jessen-Fubini theorem yields (see [9] for general theory and [11] for direct construction) that there is the unique regular probability measure $\tilde{p}$ on $B\left(R^{x}\right)$ such that $\tilde{p}(\sigma)=p_{1}\left(\sigma_{1}\right) p_{2}\left(\sigma_{2}\right) \ldots p_{n}\left(\sigma_{n}\right)$ for every cylinder set $\sigma=\sigma_{1} \times \ldots \times \sigma_{n} \times\left(\underset{k \mid n+1}{\otimes} R_{n}\right)$, where $R_{k}=R_{1}$ for $k=n+1, n+2, \ldots, \sigma_{i} \in B\left(R^{1}\right)$. The measure $\tilde{p}$ is just the countable infinite tensor power of the measure $\frac{1}{\sqrt{2 \pi}} e^{-x^{2} / 2} d x$ and we then write $\tilde{p}=\bigotimes_{n \mid 1}^{\infty} p_{n}$. Let us consider the complex Hilbert space $L^{2}(\tilde{p})$ of $B\left(R^{\infty}\right)$ measurable and $\tilde{p}$ be square summable functions on $R^{\infty}$ with the inner product

$$
(f, g)=\int_{\boldsymbol{R}^{\infty}} f(x) \overline{g(x)} d \tilde{p}_{x} .
$$

Certainly $\left(R^{\infty}, B\left(R^{\infty}\right), \tilde{p}\right)$ is a probability space and the functions $u_{k}(x)=x_{k}$ $\left(x=\left(x_{1}, x_{2}, \ldots, x_{n}, \ldots\right) \in R^{\infty}\right)$ are Gaussian independent, hence orthogonal in $L^{2}(p)$, random variables over $R^{\infty}$.

Let us fix how an $n$ and take $L^{2}\left(p_{1} \otimes \ldots \otimes p_{n}\right)$. It is plain that there is the unique unitary map $U_{n}: L^{2}\left(p_{1} \otimes \ldots \otimes p_{n}\right) \otimes L^{2}\left(\bigotimes_{k \mid n+1}^{\infty} p_{k}\right) \rightarrow L^{2}(\tilde{p})$ which maps $f_{n} \otimes g_{n}, f_{n} \in L^{2}\left(p_{1} \otimes \ldots \otimes p_{n}\right), g_{n} \in L^{2}\left(\bigotimes_{k \mid n+1}^{\infty} p_{k}\right)$ on the function $f_{n}\left(x_{1} \ldots x_{n}\right) \times$ $\times g_{n}\left(x_{n+1}, \ldots\right)=(f g)\left(x_{1}, x_{2}, \ldots, x_{n}, \ldots\right)$. Indeed, by Fubini's theorem, if $f_{n}^{\prime}, f_{n}^{\prime \prime} \in$ $L^{2}\left(p_{1} \otimes \ldots \otimes p_{n}\right), g_{n}^{\prime}, g_{n}^{\prime \prime} \in L^{2}\left(\bigotimes_{k \mid n+1}^{\infty} p_{k}\right)$, then

$$
\begin{aligned}
& \left(f_{n}^{\prime} g_{n}^{\prime}, f_{n}^{\prime \prime} g_{n}^{\prime \prime}\right)=\int_{R^{\infty}} f_{n}^{\prime} g_{n}^{\prime} \overline{f_{n}^{\prime \prime}} \overline{g_{n}^{\prime \prime}} d \tilde{p} \\
& =\int_{R^{n}} f_{n}^{\prime} \overline{f_{n}^{\prime \prime}} d\left(p_{1} \otimes \ldots \otimes p_{n}\right) \cdot \int_{R_{n+1}^{\infty}}^{\int} g_{n}^{\prime} \overline{g_{n}^{\prime \prime}} d p\left(\bigotimes_{k \mid n+1}^{\infty} p_{k}\right)
\end{aligned}
$$

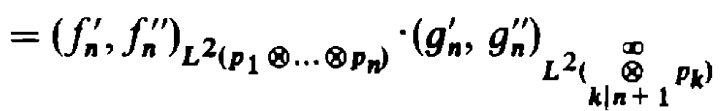

We can consequently just write the equality

$$
L^{2}(\tilde{p})=L^{2}\left(p_{1} \otimes \ldots \otimes p_{n}\right) \otimes L^{2}\left(\bigotimes_{k \mid n+1}^{\infty} p_{k}\right) .
$$


We define now on $L^{2}(\tilde{p})$ the operator $\hat{T}_{n}=\left(\bigotimes_{k \mid 1}^{n} T_{a_{k}}\right) \otimes I_{n}$, where $\bigotimes_{k \mid 1}^{n} T_{a_{k}}$ is the operator $\tilde{T}_{a}$ discussed previously for $a\left(x_{1} \ldots x_{n}\right)=\left(a_{1}\left(x_{1}\right) \ldots a_{n}\left(x_{n}\right)\right)$ and $I_{n}$ is the identity operator in $L^{2}\left(\bigotimes_{k \mid n+1}^{\infty} p_{k}\right)$. It is plain that $\left\|\hat{T}_{n}\right\|=\left\|\tilde{T}_{n}\right\|$ and $\operatorname{Sp}\left(\hat{T}_{n}\right)$ $=\operatorname{Sp}\left(\tilde{T}_{n}\right)$. On the other hand the following condition is true, see [11]:

The functions $f\left(x_{1} \ldots x_{n}\right) \in L^{2}(\tilde{p})$, where $n=1,2,3 \ldots$, i.e. cylinder functions are dense in $L^{2}(\tilde{p})$.

Since, for $k>0,\left(\tilde{T}_{n+k} f\right)\left(x_{1}, \ldots, x_{n}, x_{n+1}, \ldots\right)=f\left(a_{1}\left(x_{1}\right), a_{2}\left(x_{2}\right), \ldots, a_{n}\left(x_{n}\right)\right) \in$ $\in L^{2}(\tilde{p})$ if $f(x)=f\left(x_{1} \ldots x_{n}\right) \in L^{2}(\tilde{p})$, then having in view (3.1) and Theorem 2.0 one proves easily the following proposition:

Proposition 3.0. If $a_{k} \in A_{+}$and $\sup _{n} \prod_{k \mid 1}^{n} \frac{1}{\sqrt{s\left(a_{k}\right)}} \leqslant c<+\infty$, then the sequence $\hat{T}_{n}$ of operators in $L^{2}(\tilde{p})$ converges strongly.

When keeping in mind our previous discussion, we define formally $\hat{T}_{a}$ $=s-\lim \hat{T}_{n}=\bigotimes_{k \mid 1}^{\infty} T_{a_{k}}$. This definition would be intuitively more justified, if we could prove that for any $f \in L^{2}(\tilde{p})$,

$$
\left(\left(\bigotimes_{n \mid 1}^{\infty} T_{a_{k}}\right) f\right)\left(x_{1}, x_{2}, \ldots\right)=f\left(a_{1}\left(x_{1}\right), a_{2}\left(x_{2}\right), \ldots, a_{n}\left(x_{n}\right), \ldots\right)
$$

for $\tilde{p}$ almost all $x=\left(x_{1} \ldots x_{n} \ldots\right)$, and, moreover, that the map $a^{-1}=b$ : $\left(x_{1} \ldots x_{n} \ldots\right) \rightarrow\left(a_{1}^{-1}\left(x_{1}\right), a_{2}^{-1}\left(x_{2}\right), \ldots, a_{n}^{-1}\left(x_{n}\right), \ldots\right)$ maps $\tilde{p}$-zero sets on $\tilde{p}$-zero sets, in order to have $f(a(x))$ in $L^{2}(\tilde{p})$. Indeed, in this case the right-hand side of (3.2) would define the value of a maximal closed operator $T_{a}: f\left(x_{1}, x_{2}, \ldots\right)$ $\rightarrow f\left(a_{1}\left(x_{1}\right), a_{2}\left(x_{2}\right), \ldots\right)$ (see [5] in this matter and recall (1.2)), which coincides with the strong limit $s$-lim $\hat{T}_{n}$ on cylinder functions, and consequently $T_{a}$ itself would be a bounded operator in $L^{2}(\tilde{p})$. Our next goal is to show that this is really the case, provided the assumptions of Proposition 3.0 hold true. As easily seen, we have merely to show that if $\tilde{p}(\sigma)=0$, then $\tilde{p}\left(a^{-1}(\sigma)\right)=0$ for $\sigma \in B\left(R^{\infty}\right)$. We shall apply the Kakutani's theorem on product measures ([7], [6], p. 15). Let $\mu_{n}, v_{n}$ be two probability measures on a $\sigma$-field $B_{n}$ of subsets of $\Omega_{n}$. Let $\eta_{n}$ be a probability measure on $B_{n}$ such that $\mu_{n} \ll \eta_{n}$ and $v_{n} \ll \eta_{n}$ and define

$$
\varrho\left(\mu_{n}, v_{n}\right)=\int_{\Omega_{n}} \sqrt{\frac{d \mu_{n}}{d \eta_{n}} \frac{d v_{n}}{d \eta_{n}}} d \eta_{n},
$$

$\left(\mu_{n}, v_{n}\right)$ does not depend on the choice of $\eta_{n}$. The Kakutani's theorem says that if $\prod_{n \mid 1}^{\infty} \varrho\left(\mu_{n}, v_{n}\right)>0$, then $\bigotimes_{n \mid 1}^{\infty} \mu_{n} \equiv \bigotimes_{n \mid 1}^{\infty} v_{n}$, i.e. the countable products are mutually absolutely continuous. Suppose now that $a_{n} \in A_{+}$and $\Omega_{n}=R^{1}$, take $\mu_{n}=p_{n}$ and $v_{n}=p_{n} \circ a_{n}$ and $\eta_{n}=p_{n}$. 
It follows that $\varrho\left(p_{n}, v_{n}\right)=\frac{1}{\sqrt{2 \pi}} \int_{-\infty}^{+\infty} \sqrt{a_{n}^{\prime}(s)} e^{-\left(a_{n}(s)^{2}+s^{2}\right) / 4} d s$. Since $a_{n} \in A_{+}$ then $0<a_{n}^{\prime}(s) \leqslant q_{a_{n}} \leqslant 1$ which implies that $a_{n}(s)^{2} \leqslant q_{a_{n}}^{2}, s \leqslant s^{2}$. Since 0 $<q_{a} \leqslant 1$, i.e. $q_{a}^{2}+1 \leqslant 2$ then $a_{n}(s)^{2}+s^{2} \leqslant\left(q_{a_{n}}^{2}+1\right) s^{2} \leqslant 2 s^{2}$, i.e. $-\left(a_{n}(s)^{2}+s^{2}\right)$ $\geqslant-2 s^{2}$. It follows now that

$$
\varrho\left(p_{n}, v_{n}\right) \geqslant \frac{\sqrt{s\left(a_{n}\right)}}{\sqrt{2 \pi}} \int_{-\infty}^{+\infty} e^{-\left(a_{n}(s)^{2}+s^{2}\right) / 4} d s \geqslant \frac{\sqrt{s\left(a_{n}\right)}}{\sqrt{2 \pi}} \int_{-\infty}^{+\infty} e^{-s^{2} / 2} d s=\sqrt{s\left(a_{n}\right)} .
$$

Suppose $\sup _{n} \prod_{k \mid 1}^{n} \frac{1}{\sqrt{s\left(a_{n}\right)}}<+\infty$. Since $s\left(a_{n}\right) \leqslant 1$ then $\varrho=\prod_{k \mid 1}^{\infty} s\left(a_{k}\right)$ is finite and positive, and consequently $+\infty>\prod_{n \mid 1}^{\infty} \varrho\left(p_{n}, v_{n}\right)>0$. By Kakutani's theorem $\tilde{p}$ $=\bigotimes_{n \mid 1}^{\infty} p_{n} \equiv \bigotimes_{n \mid 1}^{\infty} p_{n} \circ a_{n} \stackrel{d}{=} \tilde{p}_{a}$. Hence, $\tilde{p}(a(\sigma))=0$ if and only if $\tilde{p}(\sigma)=0$ for $\sigma \in B\left(R^{\infty}\right)$, i.e. $\tilde{p}\left(a\left(a^{-1}(\sigma)\right)\right)=\tilde{p}(\sigma)=0$ if and only if $\tilde{p}\left(a^{-1}(\sigma)\right)=0$. This means that $\tilde{p} \equiv \tilde{p}_{a}-1$. When keeping in mind all we said above we summarize it in the following theorem:

Theorem 3.1. Assume that $a_{n} \in A_{+}$for $n=1,2,3, \ldots$ and define $a: R^{\infty}$ $\rightarrow R^{\infty}$ by the equality $a(x)=\left(a_{1}\left(x_{1}\right) \ldots a_{n}\left(x_{n}\right) \ldots\right)$ for $x=\left(x_{1}, x_{2}, \ldots, x_{n}, \ldots\right)$. Suppose that $\sup _{n} \prod_{k \mid 1}^{n} \frac{1}{\sqrt{s\left(a_{k}\right)}}<+\infty$. Then the formula $\left(T_{a} f\right)(x)=f(a(x))$ determines a well defined operator in $L^{2}\left(R^{\infty}, B\left(R^{\infty}\right), \tilde{p}\right), \quad T_{a}=\hat{T}_{a}$ and $\left\|T_{a}\right\| \leqslant \prod_{n 11}^{\infty} \frac{1}{\sqrt{s\left(a_{n}\right)}}$

4. Suppose that $a \in A_{+}$and denote by $m$ the Lebesgue measure on $R^{1}$. Let $L^{2}(m)$ stand for the complex Hilbert space of $m$-square summable functions on $(-\infty,+\infty)$. We denote by $p$ the measure $d p=\frac{1}{\sqrt{2 \pi}} e^{-x^{2} / 2} d x$ The operator $U: L^{2}(p) \rightarrow L^{2}(m)$ defined by formula $(U f)(x)=\frac{1}{\sqrt[4]{2 \pi}} f(x) e^{-x^{2} / 4}$ is unitary. This is an easy exercise to show that the operator $U T_{a} U^{-1}=S_{a}$ in $L^{2}(m)$ is defined by formula

$$
\left(S_{a} h\right)(x)=h(a(x)) e^{-\left(x^{2}-a(x)^{2}\right) / 4}
$$

for $h \in L^{2}(m)$. Let now $a$ be linear namely $a(x)=a x$ with $0<a<1$ and denote by $F$ the Fourier-Plancherel operator in $L^{2}(m)$. We define $R_{a}$ $=F S_{a} F^{-1}$. It is plain that $R_{a}$ is unitary equivalent to $T_{a}$. Let $h$ be a complex 
function on $R^{1}$ in the Schwartz space $\mathscr{S}\left(R^{1}\right)$. Recall that $F \mathscr{S}=\mathscr{S}$ and is dense in $L^{2}(m)$.

Define $g=F^{-1} h$. Then

$$
\begin{aligned}
\left(F S_{a} g\right)(x) & =\frac{1}{\sqrt{2 \pi}} \int_{-\infty}^{+\infty} e^{i x s}\left(S_{a} g\right)(s) d s \\
& =\frac{1}{\sqrt{2 \pi}} \int_{-\infty}^{+\infty} e^{i x s} g(a(s)) e^{-\left(1-a^{2}\right) s^{2} / 4} d s \\
& =\frac{1}{\sqrt{2 \pi}} \int_{-\infty}^{+\infty} e^{i x s} e^{-\left(1-a^{2}\right) s^{2} / 4}\left(F^{-1} h\right)(a s) d s \\
& =\frac{1}{\sqrt{2 \pi}} \int_{-\infty}^{+\infty} e^{i x s} e^{-\left(s^{2}-a^{2} s^{2}\right) / 4}\left\{-\frac{1}{\sqrt{2 \pi}} \int_{-\infty}^{+\infty} e^{-i a s} h(a) d u\right\} d s \\
& =\frac{1}{\sqrt{2 \pi}}\left\{\frac{1}{\sqrt{2 \pi}} \int_{-\infty}^{+\infty} e^{i(x s-a s u)} e^{-\left(1-a^{2}\right) s^{2} / 4} d s\right\} h(u) d u .
\end{aligned}
$$

Since

$$
e^{-\frac{b}{2} v^{2}}=-\frac{1}{\sqrt{2 \pi b}} \int_{-\infty}^{+\infty} e^{i \nu s} e^{-s^{2} / 2 b} d s
$$

$(b>0)$ we get that taking $b=2 /\left(1-a^{2}\right), v=x-a u$

$$
\left(F S_{a} g\right)(x)=\frac{1}{\sqrt{\left(1-a^{2}\right) \pi}} \int_{-\infty}^{+\infty} e^{-(x-a u)^{2} /\left(1-a^{2}\right)} h(u) d u .
$$

It follows that if $a(x)=a x, 0<a<1$, then

$$
\left(R_{a} h\right)(x)=\left(F S_{a} F^{-1} h\right)(x)=\frac{1}{\sqrt{\left(1-a^{2}\right) \pi}} \int_{-\infty}^{+\infty} e^{(x-a u)^{2} /\left(1-a^{2}\right)} h(u) d u
$$

We see just that in our case $T_{a}$ is unitarily equivalent to the integral operator $R_{a}$ determined by formula (4.0). If $a=1$ then $R_{a}$ is the identity operator, which simply means that if $a \rightarrow 1-$ in (4.0), then $R_{a}$ approaches identity, by simple properties of singular Poisson integral.

Suppose now that $0<a_{k}<1$ for $k=1,2, \therefore, n$ and take $a_{k}(x)=a_{k} x$. 
Then $\bigotimes_{k \mid 1}^{n} T_{a_{k}}$ is unitarily equivalent to an operator in $L^{2}\left(m^{\otimes n}\right)$ namely to $R^{a}$ $=\bigotimes_{k \mid 1}^{n} R_{a_{k}}$, defined by formula

$$
\begin{aligned}
& \left(\boldsymbol{R}^{a} f\right)\left(\tilde{x}_{1} \ldots \tilde{x}_{n}\right) \\
& =\frac{1}{\left(\prod_{k \mid 1}^{n}\left(1-a_{k}^{2}\right) \pi\right)^{1 / 2}} \int_{R^{n}} f\left(x_{1} \ldots x_{n}\right) e^{-\sum_{k \mid 1}^{n}\left(x_{k}-a_{k} x_{k}\right)^{2} /\left(1-a_{k}^{2}\right)} d x_{1} \ldots d x_{n} .
\end{aligned}
$$

It follows from Proposition 2.0 that $\left\|R^{a}\right\|=\prod_{k \mid 1}^{n} \frac{1}{\sqrt{a_{k}}}$.

5. The present section deals with the spectrum of $T_{a}$. Suppose $a(x)=a x$ and $a>0$. Then for $f(x)=x^{k}(k=1,2, \ldots)(T f)(x)=a^{k} f(x)$, i.e. $a^{k}$ are in the point spectrum of $T_{a}$. Suppose now that $z=u+i v\left(u, v\right.$ real) and define $f_{z}(x)$ $=e^{z \ln |x|}$ for $x \neq 0$. Suppose that $\operatorname{Re} z=u>0$. Since $1+\ln |x| \leqslant|x|$ for $x \neq 0$, then $\left|f_{z}(x)\right|^{2}=e^{2 \operatorname{un}|x|} \leqslant e^{2(|x|-1)}$ if $u>0$. It follows that $f_{z} \in L^{2}(p)$. On the other hand, $f_{z}(a x)=e^{z|n| a x \mid}=e^{u \ln |a|} e^{i v|n| a \mid} f_{z}(x)$. If $u$ runs over $[0,+\infty)$, then since $\ln |a|<0$, $e^{u \ln |a|}$ runs over the interval $(0,1]$. But $v$ is arbitrary. Hence the set of numbers $\xi=e^{z \ln |a|}$ for $\operatorname{Re} z>0$ covers the set $0<|\xi| \leqslant 1$. It follows that $z$ such that $\operatorname{Re} z>0$ are in the point spectrum $\operatorname{Sp}_{p}\left(T_{a}\right)$ of $T_{a}$. Notice now that our $f_{z}$ is a bounded function. This is the observation of J. Janas (private communication) that $f_{z}$ even for $u=\operatorname{Re} z>-\frac{1}{2}$ are in $L^{2}(p)$. We argue as follows: let $0>u=\operatorname{Re} z>-\frac{1}{2}$. For positive $c<d$,

$$
\int_{c}^{d}\left|f_{z}(x)\right|^{2} d x=\int_{c}^{d} e^{2 u|n| x \mid} d x=\left.\frac{x^{2 u+1}}{2 u+1}\right|_{c} ^{d} .
$$

Hence the improper Lebesgue integral $\int_{0}^{d}\left|f_{z}(x)\right|^{2} d x=d^{2 u+1 / 2 u+1}$ is finite. By a similar taken we get that, for $c<0, \int_{c}^{0}\left|f_{z}(x)\right|^{2} d x$ is also finite. Consequently $\left|f_{z}(x)\right|^{2}$ is Lebesgue summable in $[-1,+1]$. It follows that $f_{z} \in L^{2}(p)$ because $\left|f_{z}(x)\right|^{2} e^{-x^{2} / 2} \leqslant\left|f_{z}(x)\right|^{2}$ for $|x| \leqslant 1$ and $\left|f_{z}(x)\right|^{2}$ is bounded by 1 for $|x|>1$ because $u<0$. Applying now the arguments similar to that ones used previously, we conclude that $f_{z}(a x)=e^{z \ln a} f_{z}(x)$ and the numbers $\xi=e^{z \ln a}$, where $0>\operatorname{Re} z>-\frac{1}{2}$ cover the annulus $1<|\xi|<1 / \sqrt{a}$. Summing $\operatorname{Lp}\{\xi: 0<|\xi|<1 / \sqrt{a}\} \subset \operatorname{Sp}_{p}\left(T_{a}\right)$. Notice now that $T_{a}^{-1}$ exists. The range of $T_{a}$ contains all monomials $x^{k} ; k=0,1,2, \ldots$ and consequently is a dense subset of $L^{2}(p)$. It follows that $0 \in \operatorname{Sp}_{c}\left(T_{a}\right)=$ the continuous spectrum of $T_{a}$. 
Observe that the circle $C_{a}=\{\xi:|\xi|=1 / \sqrt{a}\} \subset \mathrm{Sp}_{c}(T)$. Indeed, there is no point spectrum in $C_{a}$. To see this suppose that $T_{a} f=\xi f$ for $\xi \in C_{a}$. Then

$$
\begin{aligned}
& \eta=\int_{-\infty}^{+\infty}|f(a x)|^{2} e^{-x^{2} / 2} d x=\frac{1}{a} \int_{-\infty}^{+\infty}|f(x)|^{2} e^{-x^{2} / 2} d x \\
& \text { and } \eta=\frac{1}{a} \int_{-\infty}^{+\infty}|f(x)|^{2} e^{-x^{2} / 2 a^{2}} d x
\end{aligned}
$$

and consequently $f=0$. It follows now that there is no point spectrum on $C_{a}$ of adjoint operator $T_{a}^{*}$. Hence $C_{a} \subset \mathrm{Sp}_{c}\left(T_{a}\right)$ q.e.d. We just proved the following theorem:

THEOREM 5.0. Suppose $a(x)=a x$ where $0<a<1$. Then $\operatorname{Sp}_{p}\left(T_{a}\right)=\{z: 0$ $<|z|<1 / \sqrt{a}\}, \operatorname{Sp}_{c}\left(T_{a}\right)=\{0\} \cup C_{a}$ and consequently $\operatorname{Sp}_{c}\left(T_{a}^{*}\right)=\{0\} \cup C_{a}$ and $\{z: 0<|z| 1 / \sqrt{a}\}$ equals to the residual spectrum of $T_{a}^{*}$.

The operator $T_{a}$ of our theorem is unitarily equivalent to the integral operator $R_{a}$ in $L^{2}(m)$ defined by formula

$$
\left(R_{a} k\right)(x)=-\frac{1}{\sqrt{\left(1-a^{2}\right) \pi}} \int_{-\infty}^{+\infty} e^{-(x-a u)^{2} /\left(1-a^{2}\right)} k(u) d u .
$$

It follows now from Theorem 3.0 that the following theorem holds true:

Theorem 5.1. Suppose $a(x)=a x$ where $0<a<1$. Then the integral equation

$$
\lambda f(x)=\frac{1}{\sqrt{\left(1-a^{2}\right) \pi}} \int_{-\infty}^{+\infty} e^{-(x-a u)^{2} /\left(1-a^{2}\right)} f(u) d u
$$

has non-trivial solution $f \in L^{2}(m)$ if and only if $0<|\lambda|<1 / \sqrt{a}$.

6. The space $L^{2}(p)$ where $d p=\frac{1}{\sqrt{2 \pi}} e^{-x^{2} / 2} d x$ is spanned by polynomials, i.e. the linear manifold of polynomials is dense $L^{2}(p)$. The orthonormalization procedure applied to the sequence of monomials $g_{n}(x)$ $=x^{n}, n=0,1,2, \ldots$, leads to the orthonormal basis of $L^{2}(p)$, namely to the sequence $\left\{h_{n}(x) / \sqrt{n !}\right\}, n=0,1,2, \ldots$, where $h_{n}(x)=(-1)^{n} e^{x / 2} \frac{d^{n}}{d x^{n}}\left(e^{-x^{2} / 2}\right)$ is the $n$-th Hermite polynomial for the weight function $\frac{1}{\sqrt{2 \pi}} e^{-x^{2} / 2}$. The 
generating function. for $\left\{h_{n}\right\}$ is the function $\varphi_{u}(x)=\sum_{n \mid 0}^{\infty} \frac{h_{n}(x)}{n !} u^{n}=e^{u x-u^{2} / 2}$ defined for real $x$ and arbitrary complex $u$. It is plain that $\varphi_{u} \in L^{2}(p)$ for each $u$. Moreover, the functions $\varphi_{u}$ span $L^{2}(p)$. Indeed if

$$
\left(\varphi_{u}, g\right)=\frac{1}{\sqrt{2 \pi}} \int_{-\infty}^{+\infty} e^{-x / 2} e^{u x-u^{2} / 2} \overline{g(x)} d x=0
$$

for $g \in L^{2}(p)$ and all $u \in C$, then $\int_{-\infty}^{+\infty} e^{u x} \overline{g(x)} e^{-x^{2} / 2} d x=0$ for $u \in C$. Taking $u=i s, s$ real, we infer that the Fourier transform of the $m$-summable function $\overline{g(x)} e^{-x^{2} / 2}$ vanishes. Consequently $g(x)=0 m$-a.e. which implies that $g(x)=0$ p-a.e. q.e.d.

One can get an isomorphic picture of $L^{2}(p)$ as a Hilbert space of function with a Reproducing Kernel. For references of such spaces see [1] and [8]. To begin with we notice that to every $f \in L^{2}(p)$ there corresponds the function $\hat{f}(u)=\left(f, \varphi_{u}\right)_{L^{2}(p)}$ of the complex variable $u \in C$. Since the functions $\varphi_{u}$ span $L^{2}(p)$, then $\hat{f}(u) \stackrel{g}{=}(u)$ for all $u\left(f, g \in L^{2}(p)\right)$ if and only if $f=g$. We define now the inner product $(\hat{f}, \hat{g})$ by formula $(\hat{f}, \hat{g})=(f, g)_{L^{2}(p)}$. Notice now that the correspondence between $\hat{f}$ and $f$ is linear. Let $H$ be the linear span of $\hat{f}-s$. Notice that

$$
|\hat{f}(u)| \leqslant\left\|\varphi_{u}\right\|_{L^{2}(p)}\|f\|_{L^{2}(p)} \quad \text { for } f \in L^{2}(p) .
$$

Since $\|\hat{f}\|_{H}=\|f\|_{L^{2}(p)}$ by definition, the linear space $H$ of $\hat{f}-e s$ is a Hilbert space with Reproducing Kernel equal to $\hat{\varphi}_{\bar{v}}(u)=\left(\varphi_{\bar{v}}, \varphi_{\bar{u}}\right)_{L^{2}(p)}=K(u, v)$. Indeed $(\hat{f}(\cdot), K(\cdot, v))_{H}=\left(\hat{f}, \hat{\varphi}_{\bar{v}}\right)_{H}=\left(f, \varphi_{\bar{v}}\right)_{L^{2}(p)}=\hat{f}(v)$ by definition of $\hat{f}$ and $\left({ }^{\prime},\right)_{H}$. We have now to figure out the explicit form of $K(u, v)$. By definition

$$
\begin{aligned}
K(u, v) & =\left(\varphi_{\bar{v}}, \varphi_{\bar{u}}\right)=\frac{1}{\sqrt{2 \pi}} \int_{-\infty}^{+\infty} e^{\bar{\sigma} x-\bar{v}^{2} / 2} e^{u x-u^{2} / 2} e^{-x^{2} / 2} d x \\
& =e^{-\bar{v}^{2} / 2-u^{2} / 2} \frac{1}{\sqrt{2 \pi}} \int_{-\infty}^{+\infty} e^{(\bar{v}+u) x} e^{-x^{2} / 2} d x
\end{aligned}
$$

Since $e^{b z^{2} / 2}=\frac{1}{\sqrt{2 \pi b}} \int_{-\infty}^{+\infty} e^{z x-x^{2} / 2 b} d x$ for $b>0$ and arbitrary complex $z$, then

$$
K(u, v)=e^{-\bar{v}^{2} / 2-u^{2} / 2} e^{\frac{1}{2}\left(\bar{v}^{2}+2 \bar{v} u+u^{2}\right)}=e^{u \bar{v}} .
$$


Since $\hat{f}(u)=\frac{1}{\sqrt{2 \pi}} \int_{-\infty}^{+\infty} f(x) e^{u x-u^{2} / 2} \cdot e^{-x^{2} / 2} d x$, then $f(u)$ is an entire function of $u \in C$. Moreover, since $\left\{h_{n} / \sqrt{n !}\right\}$ is an orthonormal basis in $L^{2}(p)$, and the correspondence $f \rightarrow \hat{f}$ is a unitary map in $H$, we have the formula

$$
f \rightarrow \hat{f}(u)=\left(f, \varphi_{\bar{u}}\right)_{L^{2}(p)}=\sum_{n \mid 0}^{\infty} \frac{\left(f, h_{n}\right)}{\sqrt{n !}} \frac{u^{n}}{\sqrt{n !}}=\sum_{n \mid 0}^{\infty}\left(f, \frac{h_{n}}{\sqrt{n !}}\right) \frac{u^{n}}{\sqrt{n !}}
$$

which, when taking $f=h_{n} / \sqrt{n !}$ yields, that $\left(\frac{1}{\sqrt{m !}} \hat{h}_{m}\right)(u)=\frac{u^{m}}{\sqrt{m !}} \stackrel{\mathrm{d} f}{=} w_{n}(u)$, i.e. the sequence $\left\{w_{n}(u)\right\}=\left\{u^{n} / \sqrt{n !}\right\}$ is an orthonormal basis of $H$. We know that $\hat{f}(u)$ is an entire function and

$$
f(u)=\sum_{n=0}^{\infty}\left(\hat{f}, w_{n}\right) \frac{u^{n}}{\sqrt{n !}}
$$

as well

$$
\sum_{n \mid 0}^{\infty}\left|\left(f, w_{n}\right)\right|^{2}<+\infty
$$

Conversely, if $\sum_{n \mid 0}\left|a_{n}\right|^{2}<+\infty$, then defining $f=\sum_{n \mid 0}^{\infty} a_{n} \frac{h_{n}}{\sqrt{n !}} \in L^{2}(p)$ we get that

$$
\hat{f}(u)=\sum_{n=0}^{\infty} a_{n} \frac{u^{n}}{\sqrt{n !}} \quad \text { for } u \in C
$$

It follows that $H$ consists of entire functions of form as in (6.0) and the coefficients satisfy (6.1). This means that $H$ is exactly the Bargmann space $B^{2}$, see [2], namely the Hilbert space of entire functions $\hat{f}(u)$ such that

$$
\int_{C}|\hat{f}(u)|^{2} e^{-|u|^{2}} d m_{n}<+\infty
$$

$\left(m_{n}=\right.$ the planar Lebesgue measure) with the inner product

$$
(\hat{f}, \hat{g})=\frac{1}{\pi} \int_{C} \hat{f}(u) \overline{\hat{g}(u)} e^{-|u|^{2}} d m_{n}
$$

and as we have seen with the reproducing kernel $K(u, v)=e^{u \bar{v}}$. Suppose now 
that $a \in A_{+}$and let us take the operator $T_{a}$ and its image $\hat{T}_{a}$ in the space $H=B^{2}$. It follows that $\widehat{T_{a} f}=\hat{T}_{a} \hat{f}$ for $\hat{f} \in B^{2}$ and for $u \in C$

$$
\begin{aligned}
\left(\hat{T}_{a}^{*} \hat{f}\right)(u) & =\left(\widehat{\left.T_{a}^{*} f\right)}(u)=\left(T_{a}^{*} f, \varphi_{u}\right)_{L^{2}(p)}=\left(f, T_{a} \varphi_{u}\right)_{L^{2}(p)}\right. \\
& =\frac{1}{\sqrt{2 \pi}} \int_{-\infty}^{+\infty} f(x) e^{u a(x)-u^{2} / 2-x^{2} / 2} d x \\
& =e^{-u^{2} / 2} \frac{1}{\sqrt{2 \pi}} \int_{-\infty}^{+\infty} e^{u a(x)} f(x) e^{-x^{2} / 2} d x
\end{aligned}
$$

If $a(x)=a x(0<a \leqslant 1)$, then

$$
\left(f, T_{a} \varphi_{\bar{u}}\right)=\left(f, \varphi_{a u}\right) e^{-\frac{1}{2}\left(1-a^{2}\right) u^{2}}=\hat{f}(a u) e^{-\frac{1}{2}\left(1-a^{2}\right) u^{2}}=\left(\hat{T}_{a}^{*} \hat{f}\right)(u) .
$$

This gives us the explicit formula for $\hat{T}_{a}^{*} . \hat{T}_{a}^{*}$ is obviously unitarily equivalent to $T_{a}^{*}$. Using the reproducing kernel $e^{u \bar{v}}$ we can now write

$$
\left(\hat{T}_{a}^{*} \hat{f}\right)(u)=\frac{1}{\pi} \int_{C} \hat{f}(v) e^{-\frac{1}{2}\left(1-a^{2}\right) u^{2}+\bar{v} a u-|\bar{v}|^{2}} d m_{v}
$$

The kernel $Q$ of $\hat{T}=\left(\hat{T}^{*}\right)^{*}$ is hermitian conjugate to the kernel $P(u, v)$ $=e^{\frac{1}{2}\left(1-a^{2}\right) u^{2}+\bar{v} a u}$, i.e. $Q(u, v)=\overline{P(v, u)}$. It follows that

$$
\left(\hat{T}_{a} \hat{f}\right)(u)=\frac{1}{\pi} \int_{C} \hat{f}(v) e^{-\frac{1}{2}\left(1-a^{2}\right) \bar{v}^{2}+a \bar{v} u} e^{-|\bar{v}|^{2}} d m_{v} .
$$

Formula (6.3) can be proved directly when using the equality $\left(\hat{T}_{a} \hat{f}\right)(u)$ $=\left(f, T_{a}^{*} \varphi_{a}\right),(6.2)$ and an easy computation of $\hat{\varphi}_{d}(v)=\left(\varphi_{\bar{u}}, \varphi_{\bar{v}}\right)_{L^{2}(p)}$. We see now that $\hat{T}_{a}$ and $\hat{T}_{a}^{*}$ can be figure out as an explicit integral type operators. It is plain now that we can use all the previous unitarily invariant results concerning $T_{a}, T_{a}^{*}$ with $a(x)=a x(0<a \leqslant 1)$ to the operators $\hat{T}_{a}, \hat{T}_{a}^{*}$. In particular, we can apply these ones concerning tensor products of operators in question. The infinite product can be constructed like in Section 3 using results of [3]. The suitable space will consist of entire functions of a countable number of variables.

\section{Referemes}

[1] A ronszaj in, Theory of reproducing kernels, Trans. Amer. Math. Soc. 68 (1950), 337-404.

[2] V. Bargmann, On a Hilbert space of unalytic functions and an associated integral transform, Comm. Pure Appl. Math. 14 (1961), 187-214. 
[3] V. Bargmann, Remarks on a Hilbert space of analytic functions, Proc. NAS 18 (1962), 199-204.

[4] A. Brown, C. Pearcy, Spectra of tensor products of operators, Proc. Amer. Math. Soc. 17 (1966), 162-166.

[5] N. Dunford, J. T. Schwartz, Linear Operators, Part I, New York-London 1958.

[6] T. Hida. Brownian motion, Appl. Math. 11, Springer, New York-Heidelberg.

[7] S. Kakutani, On equivalence of infinite product measures, Ann. Math. 49 (1948), 214-224.

[8] H. Meschkowski, Hilbertsche Raume mit Kernfunktion, Springer, Ser. 113, BerlinHeidelberg 1962.

[9] J. Neveu, Bases mathématiques du calcul des probabilités, Masson et CIE, Paris 1964.

[10] J. I. Petunin, A. N. Plichko, Theory of characteristics of subspaces and the applications, Kiev 1980.

[11] G. E. Shilov, Fan Dik Tin, Integral, measure and derivative on linear spaces, Moscow 1967.

INSTYTUT MATEMATYCZNY PAN, KRAKÓW

Reçu par la Rédaction le 8.08.1983 